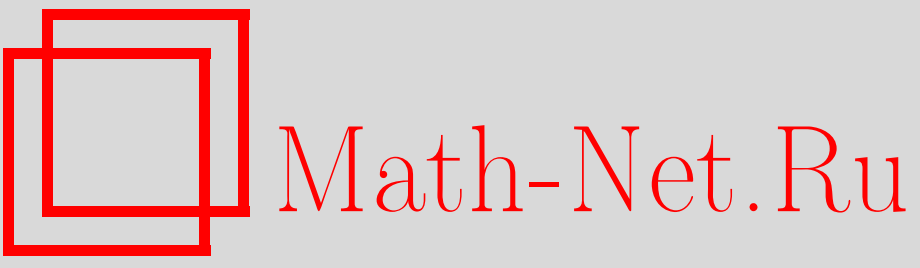

А. Ю. Плахов, А. М. Степин, Рассеяние и группы Кокстера, УМН, 1998, том 53, выпуск 2, 159-160

DOI: https://doi.org/10.4213/rm43

Использование Общероссийского математического портала Math-Net.Ru подразумевает, что вы прочитали и согласны с пользовательским соглашением

http://www.mathnet.ru/rus/agreement

Параметры загрузки:

IP : 35.173 .219 .149

26 апреля 2023 г., 05:03:37 


\title{
РАССЕЯНИЕ И ГРУППЫ КОКСТЕРА
}

\author{
А. Ю. ПлАХОВ, А. М. СТЕПИН
}

Рассматриваются гамильтоновы системы в области $\left\{x_{1}<\cdots<x_{d}\right\} \subset \mathbb{R}^{d}$ с гамильтонианом вида

$$
H=\sum_{i=1}^{d} \frac{p_{i}^{2}}{2 m_{i}}+\sum_{1 \leqslant i<j \leqslant d} u_{i j}\left(x_{j}-x_{i}\right)
$$

и билшярдные системы в некомпактных областях, обладающие рассеянием.

Будем говорить, что движение $p(t), x(t)$, а) системы с гамильтонианом (1) или б) бильярдной системы, имеет асимптотический импуль вперед $p^{+}\left(\right.$соответственно назад $\left.p^{-}\right)$, если существует предел $\lim _{t \rightarrow+\infty} p(t)=p^{+}$(соответственно $\lim _{t \rightarrow-\infty} p(t)=p^{-}$). Асимптотический импульс $\left(p^{+}\right.$или $\left.p^{-}\right)$можно рассматривать как функцию на подмножестве фазового пространства.

ПредложениЕ 1. Пусть $M^{+}{ }_{-}$мно жество асимптотических импульсов вперед тех траекторий, для которых существует и асимптотический импульс назад. Если

(2) $\quad u_{i j} \in C^{2}\left(\mathbb{R}^{+}\right), \quad \int^{\infty}\left|u_{i j}^{\prime}(x)\right| d x<\infty, \quad \lim _{x \rightarrow 0+} u_{i j}(x)=+\infty, \quad \lim _{x \rightarrow 0+} \frac{u_{i j}^{\prime}(x)}{u_{i j}(x)}=-\infty$

то $\mathrm{M}^{+}$обладает следующим свойством:

для любого вектора $v=\left(v_{1}, \ldots, v_{d}\right), v_{1}<\cdots<v_{d}$, существует $t_{0} \in \mathbb{R}^{+}$такое, ито $t v \in M^{+}$для каждого $t>t_{0}$.

Аналогичное утверждение имеет место и для множества $M^{-}$, которое получается из $M^{+}$обращением времени.

ОПРЕДЕЛЕниЕ. Рассеяние для гамильтоновых систем назовем простым, если

1. для любого вектора $v=\left(v_{1}, \ldots, v_{d}\right), v_{1}>\cdots>v_{d}$, достаточно болшшой длины (зависящей от направления $v$ ) существует такое движение, что для него $p^{-}=v$ и существует $p^{+}$;

2. На $M^{-}$существует функция $f$ такая, что $p^{+}=f\left(p^{-}\right)$.

Для бильярда в области $V$ простое рассеяние определяется аналогично со следующим изменением пункта 1: для любого $v \in \mathbb{R}^{d}$ такого, что полупрямая $t v, t>0$, пересекает $V$ по лучу, существует движение, для которого $p^{-}=v$ и существует $p^{+}$.

Системе с гамильтонианом (1) сопоставим бильярдную систему с обобщенным гамильтонианом

$$
H=\frac{1}{2} \sum_{i} p_{i}^{2}+\sum_{i<j} \delta\left(\sqrt{m_{i}} x_{i}-\sqrt{m_{j}} x_{j}\right) .
$$

Связные компоненты конфигурационного пространства этой системы суть многогранные конусы.

ПредЛОЖЕнИЕ 2. Если система с гамильтонианом (1) и условиями на потенциальнье функции (2) имеет простое рассеяние, то соответствующая бильярдная система (3) также имеет простое рассеяние.

ЗАмечАниЕ. Это предложение есть частньй случай общего утверждения о предельном поведении отображения рассеяния, когда постоянная энергии стремится к бесконечности.

Бильярды в многогранных конусах с простым рассеянием описьвает следующее утверждение.

Теорема 1. Бильярд в выпуклом коническом многограннике $V$ имеет простое рассеяние, если и только если $V$ - камера некоторой группь Кокстера.

ЗАмЕчАниЕ. Пусть $V$-конус с кусочно-гладкой границей и выпуклыми внутрь конуса гладкими компонентами границы. Тогда из простоты рассеяния биляярда в $V$ следует, что $V$-камера некоторой группы Кокстера.

Работа выполнена при поддержке Российского фонда фундаментальных исследований (гранты № 96-01-00043 и № 96-01-00713). 
Скажем, что вьпукльй многогранник в $\mathbb{R}^{d}$, содержащий некоторьй конус с непустой внутренностью, ассоциирован с группой Кокстера $W$, если линейные части отражений относительно его граней порождают $W$ и некоторый параллельный сдвиг многогранника содержится в камере группы $W$.

Теорема $1^{\prime}$. Бильярд в выпуклом многограннике $V$ с конечным числом граней имеет простое рассеяние, если и только если $V$ ассоциирован с некоторой группой Кокстера.

Из предложения 2 и теоремы 1 вьводится

ТЕорема 2. Если система с гамильтонианом (1) удовлетворяет условиям (2) и имеет простое рассеяние, то набор масс $\left\{m_{i}\right\}$ принадлежит следующему списку (с точностью до перестановки индексов $i \rightarrow n+1-i$ ):

$\left(A_{d-1}\right)$

$$
m_{i}=\frac{c}{(i-1+k)(i+k)}, \quad i=1, \ldots, d, \quad k>0
$$

в пределе $k \rightarrow \infty, \lim \left(c / k^{2}\right)=m$, получаем случай одинаковых масс $m_{i}=m$;

$\left(B_{d-1}\right) \quad m_{i}=\frac{c}{(i-1+k)(i+k)}, \quad i=1, \ldots, d-1, \quad m_{d}=\frac{c}{d-1+k}, \quad k>0$

$\left(F_{4}\right) \quad d=5, \quad m_{1}=\frac{c}{k-1}, \quad m_{2}=\frac{c}{k+1}, \quad m_{3}=\frac{c}{(k+1)\left(2 k^{-1}+1\right)}$,

$$
m_{4}=\frac{c}{2 k^{-1}+1}, \quad m_{5}=\frac{c}{2 k^{-1}-1}, \quad 1<k<2 \text {; }
$$

$\left(H_{3}\right) \quad d=4, \quad m_{1}=\frac{c}{(k-3)(k-2)}, \quad m_{2}=\frac{c}{(k-2)(k-1)}$,

$$
m_{3}=\frac{c}{(k-1) k}, \quad m_{4}=\frac{c}{k+(2-\sqrt{5}) k^{2}}, \quad 3<k<2+\sqrt{5} \text {; }
$$

$\left(H_{4}\right) \quad d=5, m_{1}=\frac{c}{(k-4)(k-3)}, \quad m_{2}=\frac{c}{(k-3)(k-2)}, \quad m_{3}=\frac{c}{(k-2)(k-1)}$,

$$
m_{4}=\frac{c}{(k-1) k}, \quad m_{5}=\frac{c}{k+(2-\sqrt{5}) k^{2}}, \quad 4<k<2+\sqrt{5}
$$

$\left(I_{2}(n)\right) \quad d=3, \quad\left(1+\frac{m_{2}}{m_{1}}\right)\left(1+\frac{m_{2}}{m_{3}}\right)=\frac{1}{\cos ^{2} \frac{\pi}{n}}, \quad n \geqslant 5 \quad$ - челое.

ЗАмечАниЕ. Аналогично (с использованием теоремы $1^{\prime}$ ) устанавливается, что для системы с гамильтонианом $H=\sum_{i} \frac{p_{i}^{2}}{2 m_{i}}+\sum_{i<j} u_{i j}\left(x_{j}-x_{i}-c_{i j}\right)$, удовлетворяющей условию (2) и имеющей простое рассеяние, набор масс принадлежит приведенному списку.

Для областей с криволинейной границей имеют место следующие утверждения.

ПредЛОЖЕниЕ 3. Пусть $V \subset \mathbb{R}^{d}$ - вьпуклая область с гладкой границей, содержащая некоторый конус с непустой внутренностью. Тогда бильярд в $V$ имеет простое рассеяние, если и только если $V$ - полупространство.

ПредложениЕ 4. Пусть $V$ - выпуклая область в $\mathbb{R}^{d}$ c кусочно-гладкой границей и трансверсальным пересечением гладжих компонент гранищы. Предположим, что число отражений бильярдной траектории от границы равномерно ограничено. Тогда бильярд в $V$ имеет простое рассеяние в том и только том случае, если V - многогранник, ассоциированный с некоторой группой Кокстера.

ПредЛОЖениЕ 6. Пусть $V \subset \mathbb{R}^{2}$ - выпуклая область, содержащая некоторый угол. Тогда бильярд в $V$ имеет простое рассеяние в том и только том случае, если V многоугольник, ассоциированный с группой Кокстера $I_{2}(n)$, действующей на плоскосmu.

Московский государственный 